\title{
Nonclassical Atomic System Dynamics Time- dependently Interacts With Finite Entangled Pair Coherent Parametric Converter Cavity Fields
}

A.B. mohamed ( $\square$ abdelbastm@aun.edu.eg )

Assiut University Faculty of Science

E. M. Khalil

Al-Azhar University

M. Y. Abd-Rabbou

Al-Azhar University

\section{Research Article}

Keywords: Time-dependent interaction, Two-mode converter, Husimi function, Squeezing phenomenon

Posted Date: February 19th, 2021

DOl: https://doi.org/10.21203/rs.3.rs-211029/v1

License: (c) (1) This work is licensed under a Creative Commons Attribution 4.0 International License.

Read Full License 


\title{
Nonclassical atomic system dynamics time-dependently interacts with finite entangled pair coherent parametric converter cavity fields
}

\author{
A.-B. A. Mohamed ${ }^{1,2} \ddagger$, E. M. Khalil ${ }^{3,4}$, M. Y. Abd-Rabbou ${ }^{4}$ \\ ${ }^{1}$ Department of Mathematics, College of Science and Humanities in Al-Aflaj, Prince \\ Sattam bin Abdulaziz University, Saudi Arabia. \\ ${ }^{2}$ Mathematics Department, Faculty of Science, Assiut University, Assiut, Egypt. \\ ${ }^{3}$ Department of Mathematics, College of Science, P.O. Box 11099, Taif University, \\ Taif 21944, Saudi Arabia. \\ ${ }^{4}$ Mathematics Department, Faculty of Science, Al-Azher University, Nassr City \\ 11884, Cairo, Egypt.
}

\begin{abstract}
We consider a time-dependent model that describes a qubit timedependently interacts with a cavity containing finite entangled pair coherent parametric converter fields. The dynamics of some quantum phenomena, as: phase space information, quantum entanglement and squeezing, are explored by atomic Husimi function, atomic Wehrl entropy, variance, and entropy squeezing. The influences of the unitary qubit-cavity interaction, the difference between the two-mode photon numbers, the initial atomic coherence, and the time-dependent qubit location are investigated. It is found that the regularity, the amplitudes and the frequency of the quantum phenomena can be controlled by the physical parameters. For the initial atomic pure state, the qubit-cavity entanglement, the qubit phase space information, and atomic squeezing can be generated strongly compared to those of the initial atomic mixed state. The time-dependent location parameters enhance the generated quantum phenomena, and its effect can be enhanced by the parameters of the two-mode photon numbers and the initial atomic coherence.
\end{abstract}

Keywords: Time-dependent interaction; Two-mode converter; Husimi function; Squeezing phenomenon.

\section{Introduction}

Probabilistic phase-space representation is a useful tool for illustrating the quantum world, it guarantees that simulated the quantum observables by the probabilistic methods [1]. The positive phase-space distribution or $Q$-Husimi function was introduced by Husimi [2], which was used for characterizing an arbitrary system of bosons and fermions $[3,4]$. Generally, the $Q$-Husimi function has widely successful and blossomed applications in quantum optics $[5,31,6]$ for studying: super fluorescence dynamics $[7,8]$, phase properties of finite states [9], and thermalization processes [10].

$\ddagger$ Corresponding author: abdelbastm@aun.edu.eg 
Based on the atomic $Q$-Husimi function, the quantum phase transition and the quantum coherence were analyzed via the Wehrl density and its entropy [11, 12]. For a single-mode atom-field interaction, the Q-Husimi function and Wehrl entropy are used as an indicator of the quantum phase space[13]. Moreover, the Wehrl entropy used as good measure for entanglement generation due to atom-field interactions $[14,15,16]$. The $Q$-Husimi function and Wehrl entropy can be applied in finding the intermode correlations in phase space $[17,18]$.

More quantum information resources were realized form qubit-cavity interaction (which is described by the popular Jaynes-Cummings model [35]) as: quantum coherence quantum correlation [19, 20, 21, 22, 23], squeezing [24], and producing quntum states [25]. Atomic squeezing phenomena of the atomic variance and the entropy squeezing [24] have more potential applications in the quantum optical measurements [26], quantum teleportation [27], and restrain the decoherence in quantum systems [28]. Atomic squeezing phenomena dynamics were investigated under the nonlinear Kerr medium [29], intrinsic decoherence [30], and parametric amplifier [31]. The generation of the entanglement was investigated under the two and three-mode Kerr nonlinear [32, 33, 34].

Quantum qubit-cavity system was described by an atomic time dependence and two-mode type of frequency converter, which can be realized in some physical systems $[36,37,38]$. Therefore, the aim of the manuscript is to finding an analytical description for the system of a qubit time-dependently interacts with a cavity contains finite pair coherent parametric converter fields when the qubit system starts with a pure/mixed state. Consequently, the dynamics of the phase space information, the quantum entanglement, and the squeezing are analyzed by the Husimi function, Wehrl entropy, variance, and entropy squeezing.

The structure of this manuscript is devoted as follows. In the next section, we describe our physical model and its analytical description. In the third section, we review the mathematical forms and the dynamics of the $Q$-Husimi function, Wehrl entropy, variance squeezing, and entropy squeezing. Finally, we conclude our results in the forth section.

\section{Physical model and its solution}

Here, we introduce the system Hamiltonian that describes a time-dependent two-level atom (qubit system) interacts with two modes of electromagnetic fields in the frame converter-type. The qubit-cavity field Hamiltonian is given by,

$$
H=\omega_{1} \hat{a}_{1}^{\dagger} \hat{a}_{1}+\omega_{2} \hat{a}_{1}^{\dagger} \hat{a}_{1}+\frac{1}{2} \omega_{0} \hat{S}_{z}+\hat{S}_{+} \hat{a}_{1}^{\dagger} \hat{a}_{2} A(t)+\hat{a}_{1} \hat{a}_{2}^{\dagger} \hat{S}_{-} A^{*}(t)
$$

$\hat{a}_{i}$ and $\hat{a}_{i}^{\dagger}$ are annihilation and creation two-mode parametric converter cavity operators, the mean photon number of $i$-mode cavity field is denoted by: $\hat{n}_{i}=\hat{a}_{i}^{\dagger} \hat{a}_{i}$. $\omega_{i}$ and $\omega_{0}$ represent the qubit and cavity frequencies, respectively. But $\hat{S}_{+}, \hat{S}_{-}$and $\hat{S}_{z}$ are the qubit Pauli matrix, which satisfy the commutations relations $\left[\hat{S}_{z}, \hat{S}_{ \pm}\right]= \pm 2 \hat{S}_{ \pm}$and $\left[\hat{S}_{+}, \hat{S}_{-}\right]=\hat{S}_{z} . \quad A(t)=(\cos p(t)+i \sin p(t)) f(t), f(t)$ is the qubit-cavity interaction 
coupling constant, and the time-dependent qubit-cavity phase is described by $p(t)$.

The motion constants are calculated by the Heisenberg equation, consequently, based on the system Hamiltonian of Eq.1, the motion equations of the qubit and cavity operators are:

$$
\begin{aligned}
& \frac{d}{d t} \hat{S}_{z}=-2 i\left(\hat{S}_{+} \hat{a}_{1}^{\dagger} \hat{a}_{2} A(t)-\hat{a}_{1} \hat{a}_{2}^{\dagger} A^{*}(t) \hat{S}_{-}\right), \\
& \frac{d}{d t} \hat{n}_{j}=-i(-1)^{j+1}\left(\hat{S}_{+} \hat{a}_{1}^{\dagger} \hat{a}_{2} A(t)-\hat{a}_{1} \hat{a}_{2}^{\dagger} A^{*}(t) \hat{S}_{-}\right), \quad j=1,2 .
\end{aligned}
$$

Then, the constant-motion operators are given by

$$
\begin{aligned}
& N_{j}=\hat{n}_{j}+\frac{1}{2}(-1)^{j} \hat{S}_{z}, j=1,2 . \\
& \hat{Q}=\hat{n}_{1}+\hat{n}_{2} .
\end{aligned}
$$

Therefore, the qubit-cavity Hamiltonian can be rewritten as

$$
\hat{H}=\omega_{1} N_{1}+\omega_{2} N_{2}+\frac{\Delta}{2} \hat{S}_{z}+\hat{S}_{+} \hat{a}_{1}^{\dagger} \hat{a}_{2} A(t)+\hat{a}_{1} \hat{a}_{2}^{\dagger} \hat{S}_{-} A^{*}(t)
$$

where $\delta=\left(\Omega_{0}-\omega_{1}-\omega_{2}\right)$

Here, the dynamical evolution of the qubit-cavity interaction of the wave-function is governed by the Schrödinger equation,

$$
i \frac{\partial}{\partial t}|\psi(t)\rangle=\hat{H}|\psi(t)\rangle
$$

To find a particular wave function, we assume that the initial state of the qubit is in a superposition from the upper $|1\rangle$ and down $|0\rangle$ states as:

$$
|S(0)\rangle_{A}=\cos \varpi|1\rangle+\sin \varpi|0\rangle .
$$

The two-mode fields are in finite entangled pair coherent states [39],

$$
\begin{aligned}
|S(0)\rangle_{f} & =\sum_{n=0}^{q} P_{n}(\xi)|q-n, n\rangle, \\
P_{n}(\xi) & =\frac{1}{\sqrt{{ }_{1} F_{0}\left(-q,-|\xi|^{2}\right)}} \frac{\xi^{n}}{\sqrt{n !(q-n) !}}=\frac{1}{\sqrt{\sum_{n=0}^{q} \frac{|\xi|^{2 n}}{n !(q-n) !}}} \frac{\xi^{n}}{\sqrt{n !(q-n) !}}
\end{aligned}
$$

where ${ }_{1} F_{0}$ is a generalized hypergeometric function, and $q$ is the difference between the two-mode of the photon numbers $q=\hat{n}_{1}-\hat{n}_{2}$. Consequently, the dynamics of the wave function can be formulated as

$$
|\psi(t)\rangle=\sum_{n=0}^{q} P_{n}(\xi)\left\{C_{1}|q-n, n, 1\rangle+C_{2}|q-n+1, n+1,0\rangle\right\},
$$

By using Eq.5, the dynamics of the wave-function coefficients $C_{i}$ are calculated by

$$
\begin{aligned}
i \frac{\partial}{\partial t} C_{1} & =\frac{1}{2} \delta C_{1}+\mu_{n} C_{2} A(t), \\
i \frac{\partial}{\partial t} C_{2} & =-\frac{1}{2} \delta C_{2}+\mu_{n} C_{1} A^{*}(t),
\end{aligned}
$$


where $\mu_{n}=\sqrt{(q-n)(n+1)}$. By consider the integrability condition is $\dot{\chi}(t)=c f(t)$ with constant $c$, the analytical solutions of the coefficients $C_{i}(10)$ are given by

$$
\begin{aligned}
C_{1}= & \left\{\cos \varpi-i \frac{1}{\tilde{M}}\left(\sqrt{M} \sin \varpi+\frac{1}{2} \cos \varpi\right) \tan V(t) \tilde{M}\right\} \\
& \times \cos (V(t) \tilde{M}) \exp \left\{-\frac{i}{2}(\delta t-V(t))\right\},
\end{aligned}
$$

and

$$
\begin{aligned}
C_{2}= & \left\{\sin \varpi+i \frac{1}{\tilde{M}}\left(\frac{1}{2} \sin \varpi-\sqrt{M} \cos \varpi\right) \tan V(t) \tilde{M}\right\} \\
& \times \cos (V(t) \tilde{M}) \exp \left\{\frac{i}{2}(\delta t-V(t))\right\} .
\end{aligned}
$$

Where $V(t)=p(t)-\delta t, M=\frac{\mu_{n}^{2}}{c^{2}}, \tilde{M}=\sqrt{\left(M+\frac{1}{4}\right.}$ and the time-dependent location of the qubit in the cavity is controlled by

$$
V(t)=\int_{0}^{t} \cos (\alpha x+\beta) d x
$$

where $\alpha$ and $\beta$ represent the time-dependent location parameters.

\section{Quantum effect dynamics}

By using the analytical solution that describes the time-dependent evaluation of the interaction between the qubit and the two-mode cavity field, we investigate the dynamics of different quantum quantifiers as $Q$-Husimi function, Wehrl entropy, variance, and entropy squeezing.

\subsection{Q-Husimi function}

Atomic coherent states $|\theta, \phi\rangle$ based on the standard angular momentum with spin- $j$ can be reconstructed as $[40,41]$,

$$
|\theta, \phi\rangle=\sum_{n=-j}^{n=j} \frac{e^{i(j-n) \phi}}{2^{j}} \sqrt{\frac{(2 j) !}{(j-n) !(j+n) !}} \sin ^{j} \theta \cot ^{n} \frac{\theta}{2}|j, n\rangle .
$$

For the two-denominational spin- $\frac{1}{2}$ (qubit), one can immediately get the atomic coherent state $|\Lambda\rangle$, as follows:

$$
|\Lambda\rangle=\cos \frac{\theta}{2}\left|1_{A}\right\rangle+\sin \frac{\theta}{2} e^{i \phi}\left|0_{A}\right\rangle .
$$

where the distribution angles $\theta$, and $\phi$ represent the atomic phase space parameters.

Beyond that, the information loss in the qubit subsystem is obtained via the atomic family of the $Q$-Husimi function, which is defined as [2],

$$
Q(\Lambda, t)=\frac{1}{2 \pi}\left\langle\Lambda\left|\rho^{A}(t)\right| \Lambda\right\rangle .
$$


On the other hand, the atomic $Q$-Husimi function can quantify the evolution of quantum entanglement by the Wehrl entropy, it is formalized as [42, 43],

$$
S(t)=\int_{0}^{2 \pi} \int_{0}^{\pi} \mathcal{D}(\Lambda, t) d \Lambda
$$

where $d \Lambda=\sin \theta d \theta d \phi$. The function $\mathcal{D}(\Lambda, t)$ is the Wehrl density, it is established in terms of the $Q$-Husimi function as,

$$
\mathcal{D}(\Lambda, t)=Q(\Lambda, t) \ln \left(\frac{1}{Q(\Lambda, t)}\right) .
$$

In the following, we quantify the dynamics of phase space atomic information and the evolution of mixedness through the $Q$-Husimi function and the Wehrl entropy, respectively.
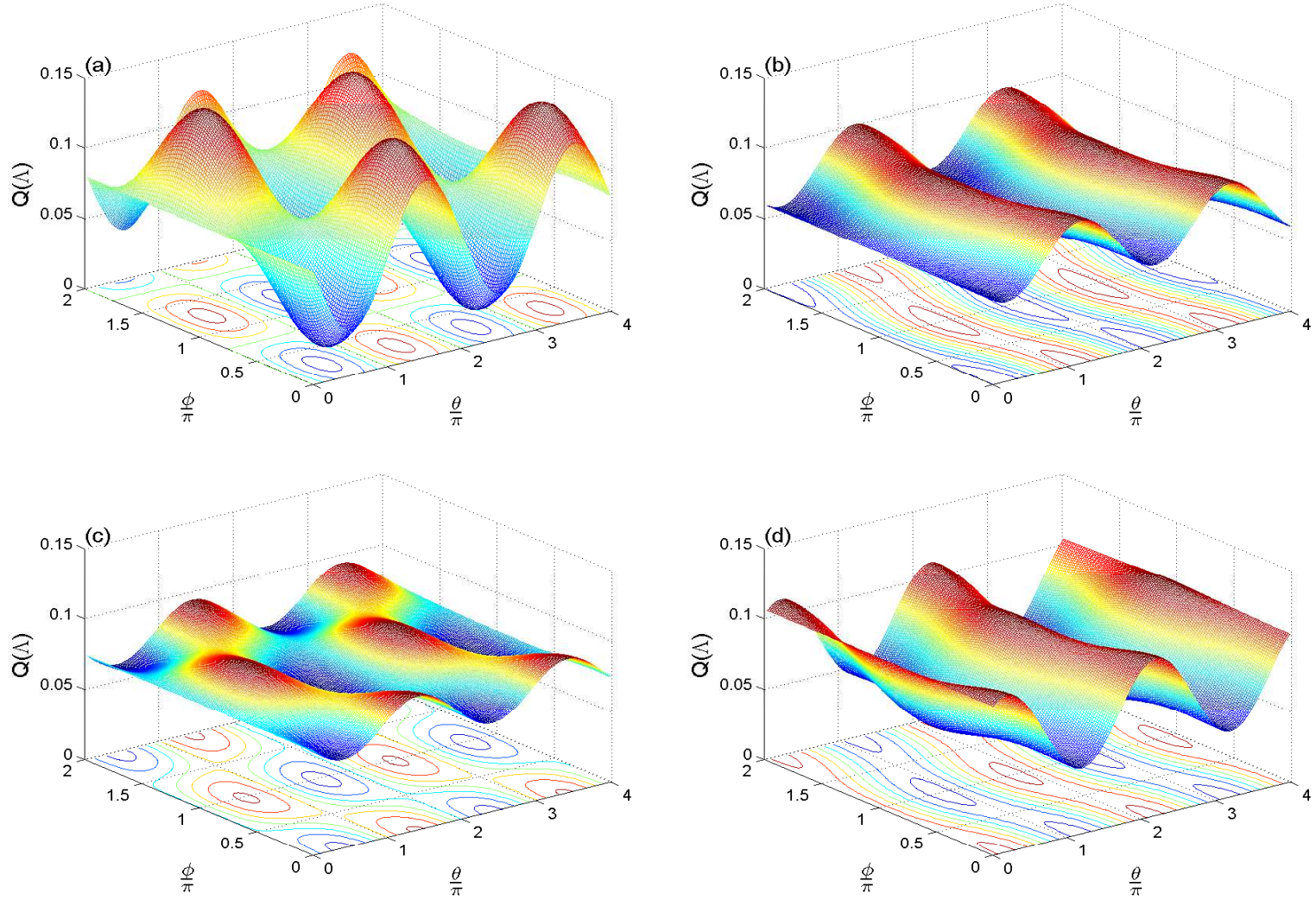

Figure 1. The $Q$-Husimi function at $\lambda t=0.6 \pi$ (that corresponds to a WE minimum value) in (a) and at $\lambda t=\pi$ (that corresponds to a WE maximum value) in (b) for $\xi=2, q=40$, and $\alpha=\beta=0$ with $\varpi=\pi$. In (c) and (d), we take the same data of (a,b) but for $q=10$.

Fig. (1) display the atomic Q-Husimi function distribution in the chosen phase space $\theta \in[0,4 \pi]$ and $\phi \in[0,2 \pi]$ at some specific values of scaled time $\lambda t$, and the variables $\alpha, \beta, q, \xi$. We assume that the qubit is initially in an pure state, while the field subsystem is initially in the finite entangled pair coherent states dependent on the 
parameter $q$. At $\lambda t=0.6 \pi$ with large values of the parameter $q$ and $\alpha=\beta=0$, the $Q$-Husimi function has periodical peaks and bottoms, the peaks occur at the points $(\phi, \theta)=\left(\frac{(4 n-1) \pi}{2}, \frac{(4 n-1) \pi}{2}\right)$, while the bottoms occur at $(\phi, \theta)=\left(\frac{(4 n-3) \pi}{2}, \frac{(4 n-3) \pi}{2}\right)$, where $n$ is set to be a positive number. By putting $\lambda t=\pi$, the peak amplitudes are decreased slightly and the bottoms are deteriorated. The $Q$-Husimi function becomes a waveform propagating in the $\theta$-axis direction. For small values of the parameter $q$, the amplitude of the fluctuations of the Husimi distribution are decreased, see Fig.(1.c). At $\lambda t=\pi$, the dependence of the Husimi distribution on the $\theta$ parameter is more than on the $\phi$ parameter, see Fig.(1.d).

Fig(2) exhibits the temporal evolution of the $Q$-Husimi distribution with the initial
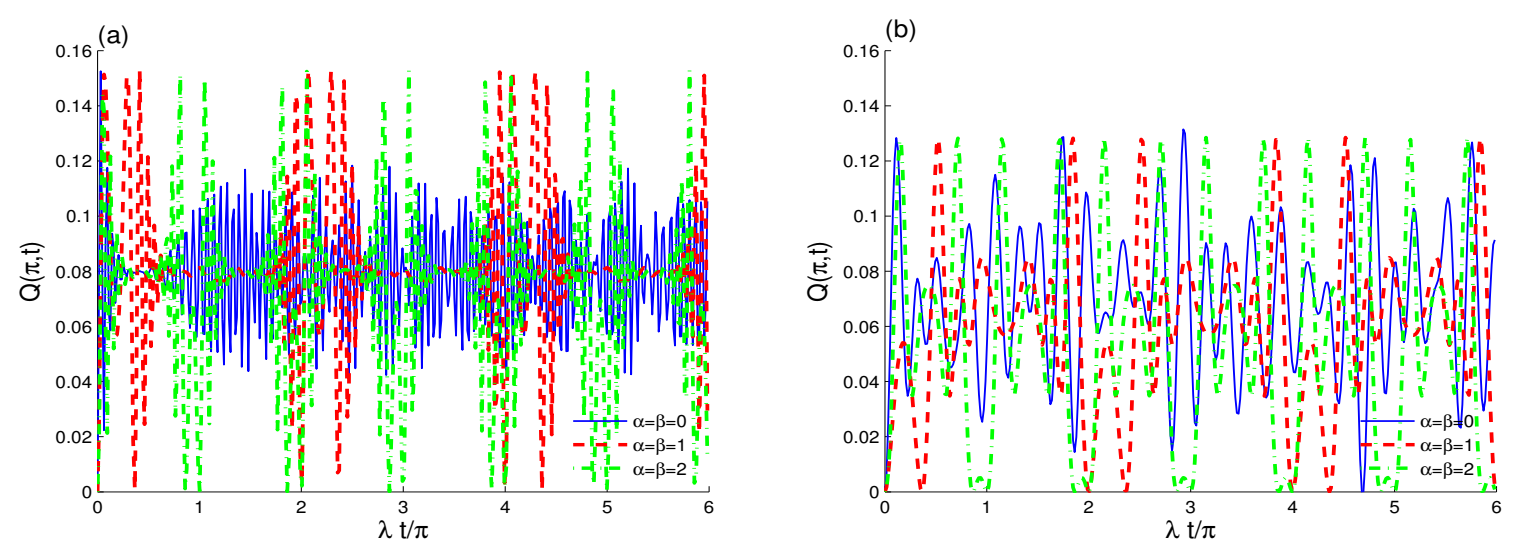

Figure 2. Time evolution of $Q(\pi, t)$ for $\xi=2, \varpi=\pi$ and the different values of $(\alpha, \beta)$ with $q=40$ in (a) and $q=10$ in (b).

pure state, where we set $q=40$. The oscillations of the Q-Husimi function depend on the value of the time-dependent parameters, where the phase space information is very delicate to time-dependent parameters. Also, one can see that the oscillations collapse in the period corresponding to the bottom of $Q(\Lambda, t)$, while the oscillations increase in the period corresponding to the peak compared to the previous Fig.(1,2.a). The behavior of $Q(\Lambda, t)$ at a specific small value of $q=10$ is displayed in Fig.(2.b). It shows that, the regular form of the phase space information got random, and the collapse intervals disappear. Nevertheless, as one increases the time-dependent parameters $(\alpha, \beta)$, the phase space information is oscillated between its maximum and zero values.

Fig. (3) esteems the degree of entanglement between the qubit subsystem and the nonlinear cavity filed by using the atomic Wehrl entropy. Initially, we assume that the initial qubit subsystem in the pure state $\varpi=\pi$, with large values of $q$. In the absence of the qubit time-dependent location, the quantum entanglement is generated between the two-mode field and the qubit subsystem. Where, a weak entanglement is generated at the collapse intervals of the $Q$-Husimi function, and a strong entanglement is generated at revival periods of the $Q$-Husimi function. Hence, there is a strong relation between the information of the phase space and the entanglement. Moreover, the strength of 

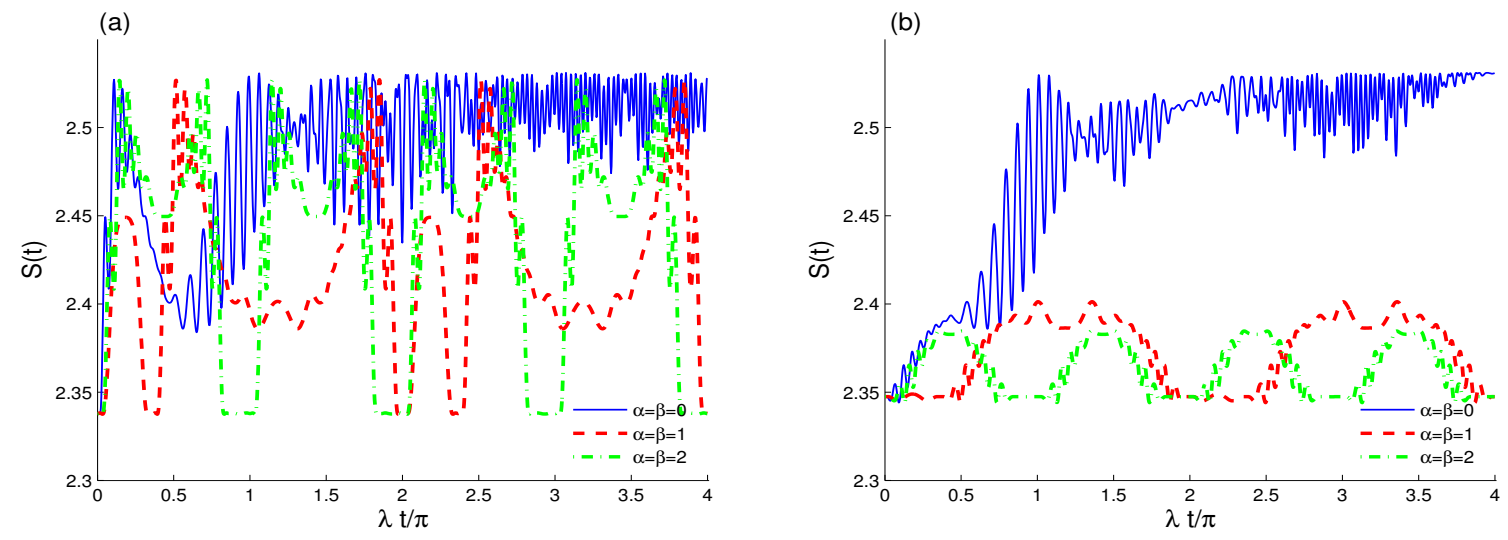

Figure 3. Dynamics of entropy $S(t)$ for $\xi=2, q=40$ and the different values of $(\alpha, \beta)$ with $\varpi=\pi$ in (a) and $\varpi=\frac{\pi}{4}$ in (b).

the time-dependent parameters leads to generate an entanglement suffers from sudden death and sudden birth $[44,45]$, this entanglement decreases as the time-dependent parameters increase. For the initial mixed state $\left(\varpi=\frac{\pi}{4}\right)$, Fig. (3.b) shows that a quantum entanglement is generated between the two subsystem. This entanglement reaches to its maximum value at $\lambda t=\pi$, followed by oscillations with a decrease in the amplitude of the oscillations. However, the time-dependent location parameters $(\alpha, \beta)$ led to the death of the entanglement at some intervals.

On the other hand, by regulate the system in the excited pure state, with $q=40$, Fig.(4.a) shows that a partial entanglement is generated with a lower intensity of oscillations. Meanwhile, the time-dependent parameters may play as a control parameter to restrain the separability behaviour of the system, and keep the survival of the entanglement. By setting up the qubit system with a mixed state $\left(\varpi=\frac{\pi}{4}\right)$, the minimum bounds of the function $S(t)$ are increased. Therefore, a strong partial entanglement is generated, it increases as the strength of time-dependent location increase.
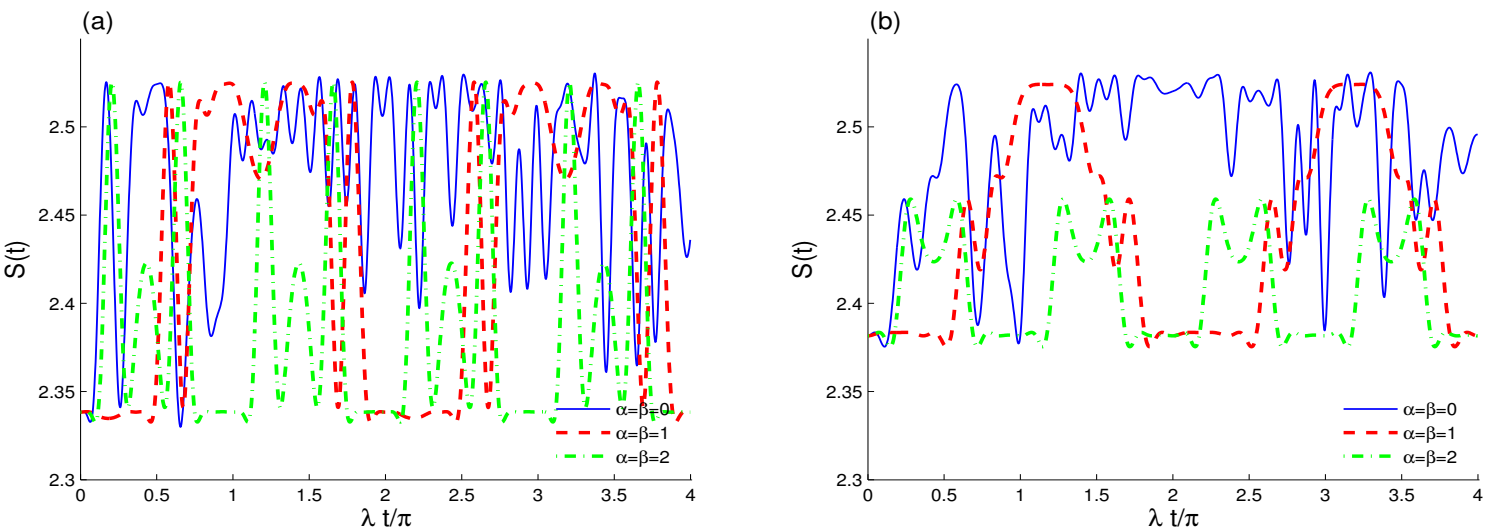

Figure 4. The same as in Fig.3 but for $q=10$. 


\subsection{Atomic squeezing}

The atomic variance is a good quantifier that takes our attention for studying the qubit squeezing dynamics, it can be formulated based on the Heisenberg uncertainty relation via the Pauli spin operators as follows,

$$
V_{l}=\frac{1}{2}\left[\sqrt{1-\left\langle\hat{\sigma}_{l}\right\rangle^{2}}-\sqrt{\left|\left\langle\hat{\sigma}_{z}\right\rangle\right|}\right] ; \quad l=x, y,
$$

where, we predict that the system is squeezed if $V_{l}<0$. Fig.(5) displays the function $V_{y}$ for a pure qubit system. For large values of $q$, the squeezing interval is achieved at the onset qubit-cavity interaction for a short period of the time. By appending the time dependence of the qubit location, the squeezing periods are repeated periodically each $\pi$. These squeezing periods have more pronounced by increasing the coefficients $(\alpha, \beta)$. The intensity of oscillations of the function $V_{y}(t)$ decreases and the periods of squeezing increase after considering the small values of the parameter $q$. We can deduce that the atomic squeezing can be increased due to the time-dependent qubit location.
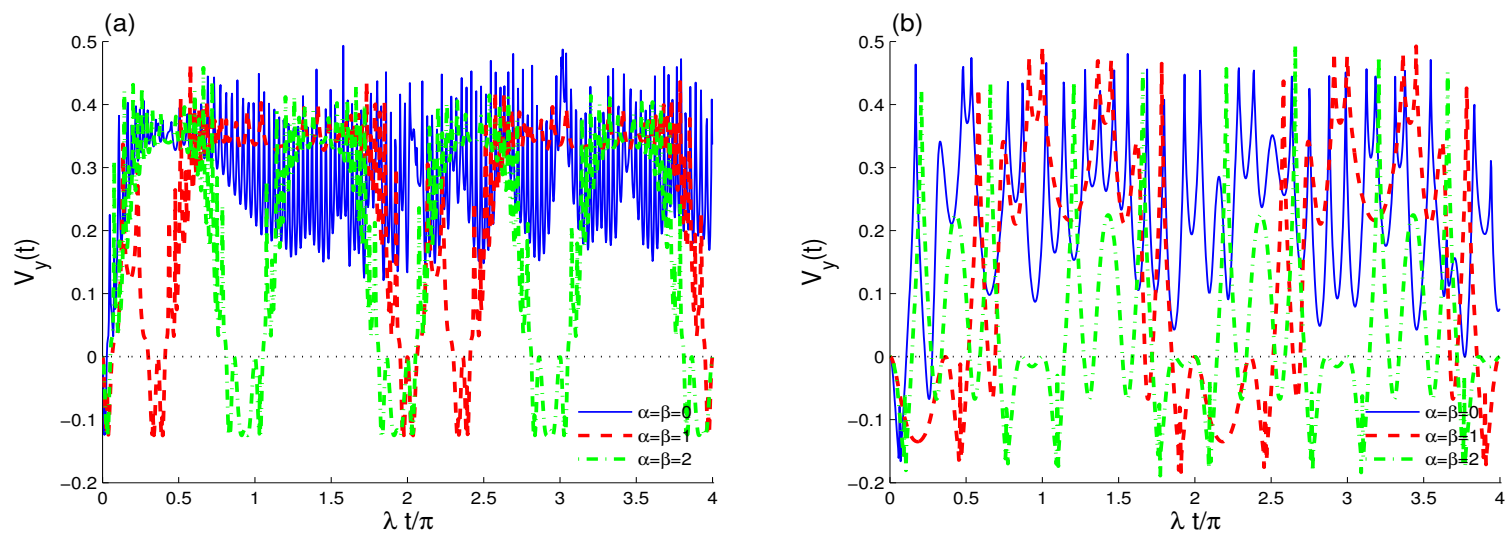

Figure 5. The variance squeezing $V_{y}(\mathrm{t})$ for $\xi=2, \varpi=\pi$ and the different values of $(\alpha, \beta)$ with $q=40$ in (a) and $q=10$ in (b).

\subsection{Entropy Squeezing:}

Finally,discuss the information of the atomic system via the entropy squeezing. By employing the Pauli spin measurements $\sigma_{l}$ in the discrete entropic uncertainty inequality, one can obtain the entropy squeezing as [24],

$$
E_{l}=\delta H\left(\sigma_{l}\right)-\frac{2}{\sqrt{\delta H\left(\sigma_{z}\right)}}<0, \quad l \equiv x \text { or } y .
$$

where $\delta H\left(\sigma_{\alpha}\right) \equiv \exp \left[H\left(\sigma_{\alpha}\right)\right]$, and it immediately satisfies the inequality

$$
\delta H\left(\sigma_{x}\right) \delta H\left(\sigma_{y}\right) \delta H\left(\sigma_{z}\right) \geq 4,
$$

With the discrete Shannon entropy,

$$
H\left(\sigma_{l}\right)=-\frac{1}{2} \sum_{i=1,2}\left[1+(-1)^{i}\left\langle\sigma_{l}\right\rangle\right] \ln \frac{1}{2}\left[1+(-1)^{i}\left\langle\sigma_{l}\right\rangle\right] .
$$



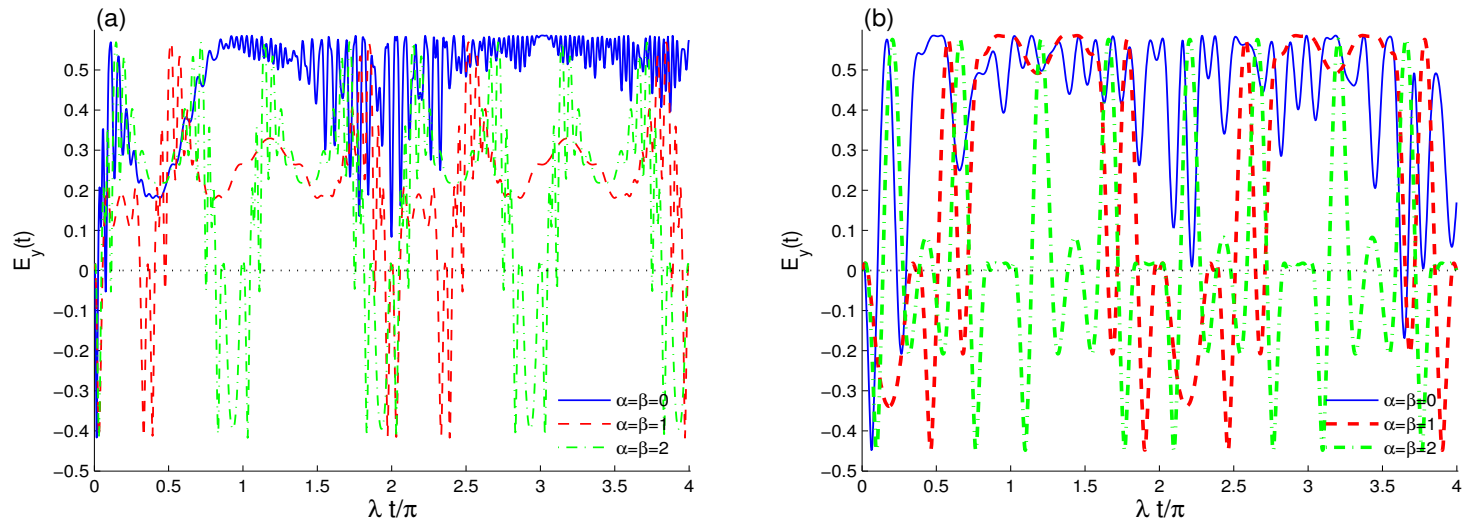

Figure 6. Dynamics of the entropy squeezing $E_{y}(\mathrm{t})$ for $\xi=2, \varpi=\pi$ and the different values of $(\alpha, \beta)$ with $q=40$ in (a) and $q=10$ in (b).

We employ the entropy squeezing $E_{y}(t)$ to analyze the generated atomic squeezing due the qubit-cavity interaction (1). Fig.(6) displays the function $E_{y}(t)$ with the large values of $q$ and the initial qubit pure state $(\varpi=\pi)$. The squeezing periods only appear after the onset of interaction, then they disappear with the the increase of the time. After adding the time-dependent location parameters, the minima of the squeezing amplitudes are increased with more oscillations. Fig.(6.b) shows that, for the small values of $q$, the squeezing periods are very sensitive for the qubit time-dependent location. The squeezing periods may be appeared again during other time intervals in the absence of the time-dependent parameters. Different behavior is shown in Fig.(7) when the qubit system starts with a mixed state, where the large values of $q$ decrease the periods of squeezing as well as the minima of the function $E_{y}(t)$. These periods increase by taking the time dependence into account. On the other hand, for a small value of $q$, the squeezing periods are vanished with different time-dependent parameters.
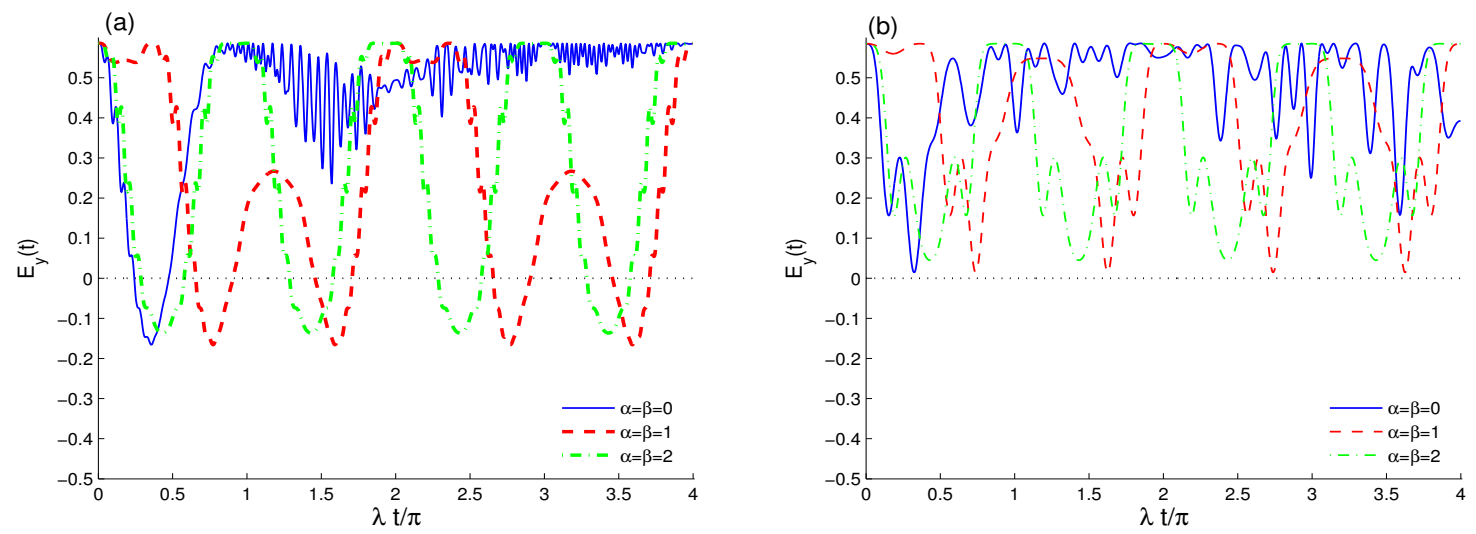

Figure 7. The same as in Fig.3 but for $\varpi=\frac{\pi}{4}$. 


\section{Conclusions}

In this paper, we have obtained an analytical solution for a time-dependent qubit that interacts with a nonlinear two-mode parametric converter cavity when the field is initially in finite entangled pair coherent states. We have investigated the dynamics of the atomic phase space non-classicality of the Q-Husimi distribution, the Wehrl entropy entanglement as well as the atomic squeezing of the variance and the entropy squeezing. The regularity and the amplitudes of the atomic phase space non-classicality oscillations depend on the diffidence between the two-mode photon numbers and the time-dependent qubit location. For the initial pure atomic state and large diffidence between the two-mode photon numbers, a strong irregular entanglement between the qubit of the two-mode cavity fields is generated, due to their interactions, with high oscillations. The period and the intensity of the generated regular oscillatory behaviour (due to the time-dependent location) of the entanglement con be controlled by the timedependent qubit location parameters. For the initially mixed state, the ability of the qubit-cavity interaction on the generation of the entanglement is lower than that of the initial pure state. The results depicted a strong relationship between the entanglement and the Q-Husimi function. Moreover, the squeezing periods are displayed by the variance and the entropy squeezing. For large values of the difference between the two-mode field and the initial pure state, the minima of atomic squeezing periods are stronger than those of the initial mixed state. The squeezing periods are very sensitive

to the time-dependent location, where the increase of the time-dependent parameters enhances the generated squeezing periods. Compliance with ethical standards

Disclosures: The authors declare no conflicts of interest.

Funding: This research received no external funding.

\section{References}

[1] Kilmov, A. B., and Sergei M. Chumakov. " A group-theoretical approach to quantum optics: models of atom-field interactions". John Wiley Sons, (2009)

[2] Husimi, K. Some properties of the Husimi function. Proc. Phys. Math. Soc. Jpn. 22, (1940), 264.

[3] Agarwal, G. S. State reconstruction for a collection of two-level systems. Phys. Rev. A 57, (1998), 1.

[4] Laura, E. C.; Rosales-Zárate; Drummond, P. D. Probabilistic Q-function distributions in fermionic phase-space. Now j. Phys.17, (2015), 032002.

[5] Hessian, H. A.; Mohamed, A.-B. A.; Quasi-Probability Distribution Functions for a Single Trapped Ion Interacting with a Mixed Laser Field. Laser Physics, 18 (2008) 1217.

[6] Mohamed, A.-B. A.; Eleuch, H.; Obada, A.-S.F. Quantum effects in two-qubit systems interacting with two-mode fields: Dissipation and dipole-dipole interplay effects. Results in Phys. 17, (2020), 103019.

[7] Lee, T. C. $Q$ representation of the atomic coherent states and the origin of fluctuations in superfluorescence. Phys. Rev. A 30, (1984), 3308. 
[8] Altland, A. ; Haake, F. Equilibration and macroscopic quantum fluctuations in the Dicke model. Now j. Phys. 14, (2012), 073011.

[9] Barnett, S. M.; Vaccaro, J. A. The Quantum Phase Operator: a Review. (London: Taylor and Francis 2007).

[10] Altland, A. ; Haake, F. Quantum Chaos and Effective Thermalization . Phys. Rev. Lett. 108, (2012), 073601.

[11] Wehrl, A. On the relation between classical and quantum-mechanical entropy. Rep. Math. Phys. 16, (1979), 353.

[12] Bužek, V., Christoph H. Keitel, and Peter L. Knight. "Sampling entropies and operational phasespace measurement. II. Detection of quantum coherences." Phys. Rev. Lett. 51, (1995) : 2594.

[13] Romera, E., del Real, R., Calixto, M. "Husimi distribution and phase-space analysis of a Dickemodel quantum phase transition". Phys. Rev. A 85 (2012) : 053831.

[14] El-Orany, F. A. "Marginal and density atomic Wehrl entropies for the Jaynes-Cummings model". j. Phys. A 41, (2008), 035303.

[15] Abdalla, M. S.; Obada, A.-S. F.; Khalil, E. M.; Ali, S. I. "The influence of phase damping on a two-level atom in the presence of the classical laser field". Laser Phys., 23, (2013), 115201.

[16] Mohamed, A. B. A.; Eleuch, H.; "Wehrl information and mixedness of a Cooper-pair box coupled to a cavity in a thermal reservoir". J Korean Phys. Soc., 68, (2016) , 513-519.

[17] Piatek, K., and W. Leonski. "Wehrl's entropy and a measure of intermode correlations in phase space." J. Phys. A, 34, (2001) : 4951.

[18] Miranowicz, A., Bajer, J., Wahiddin, M. R. B., Imoto, N. "Wehrl's entropy and a measure of intermode correlations in phase space." J. Phys. A, 34, (2001): 4951.

[19] A.-B. A. Mohamed, H. Eleuch, C. H. Raymond Ooi, Quantum coherence and entanglement partitions for two driven quantum dots inside a coherent micro cavity. Phys. Lett. A 383,(2019): 125905.

[20] A.-S. F. Obada, H. A. Hessian, A.-B. A. Mohamed, and A. H. Homid, J. Opt. Soc. Am. B 30, (2013): 1178.

[21] A.-B. A. Mohamed, H. Eleuch, C. H. Raymond Ooi, Non-locality Correlation in Two Driven Qubits Inside an Open Coherent Cavity: Trace Norm Distance and Maximum Bell Function, Sci. Rep. 9, (2019): 19632.

[22] Mishra, U., Prabhu, R. and Rakshit, D. Quantum correlations in periodically driven spin chains: Revivals and steady-state properties. Journal of Magnetism and Magnetic Materials 491, (2019): 165546.

[23] Fumania, F. K., Beradze, B., Nemati, S., Mahdavifar, S. and Japarid, G. I. Quantum correlations in the spin-1/2 Heisenberg XXZ chain with modulated Dzyaloshinskii-Moriya interaction, Journal of Magnetism and Magnetic Materials 518, (2021): 167411.

[24] Fang, M. F.; Zhou, P.; Swain, S. "Entropy squeezing for a two-level atom". J. Mod. Opt 47, (2000), 1043.

[25] Leonski, W., and R. Tanas. "Possibility of producing the one-photon state in a kicked cavity with a nonlinear Kerr medium." Phy. Rev A, 49, (1994) : R20.

[26] Sorensen, A.; Molmer, K. Phys. Lett. A Spin-Spin Interaction and Spin Squeezing in an Optical Lattice. 83, (1999), 2274.

[27] Furusawa, A.; SØrensen, J.L.; Braunstein, S.L.; Fuchs, C.A.; Kimble, H.J.; Polzik, E.S. Unconditional Quantum Teleportation. Science 282, (1998), 706.

[28] Wineland, D. J., Bollinger, J. J., Itano, W. M., Heinzen, D. J. "Squeezed atomic states and projection noise in spectroscopy", Phys. Rev. A, 50, (1994): 67.

[29] Baghshahi, H. R., Tavassoly, M. K., Behjat, A. "Entropy squeezing and atomic inversion in the k-photon Jaynes-Cummings model in the presence of the Stark shift and a Kerr medium: A full nonlinear approach", Chinese Phys. B, 23, (2014): 074203.

[30] Obada, A.-S. F., Khalil, E. M., Ahmed, M. M. A., Elmalky, M. M. Y. Influence of an External Classical Field on the Interaction Between a Field and an Atom in Presence of Intrinsic 
Damping", Int. J. Theor. Phys., 57, (2018) : 2787-2801.

[31] Khalil, E. M., Abdalla, M. S., Obada, A. F., Perina, J. "Entropic uncertainty in two two-level atoms interacting with a cavity field in presence of degenerate parametric amplifier", JOSA B, 27, (2010): 266-276.

[32] Leonski, W., and A. Miranowicz. "Kerr nonlinear coupler and entanglement." J. Optics B, 6, (2004) : S37.

[33] Kalaga, J. K., W. Leonski, and R. Szczenniak. "Quantum steering and entanglement in threemode triangle Bose-Hubbard system." Quantum Information Processing, 16, (2017) : 265.

[34] Kowalewska-Kudlaszyk, A., W. Leonski, and J. Perina Jr. "Generalized Bell states generation in a parametrically excited nonlinear coupler". Phys. Scr. T147 (2012): 014016.

[35] E. T. Jaynes and F. W. Cummings, Proc. IEEE 51, (1963) 89-109 .

[36] Louisell, W. H. "Coupled mode and parametric electronics". Wiley (1960).

[37] Khalil, E. M., Abdalla M.S., and Obada A.-S.F. "Entropy and variance squeezing of two coupled modes interacting with a two-level atom: Frequency converter type." Annals of Physics, $\mathbf{3 2 1 m}$ (2006): 421-434.

[38] Mohamed, A.-B.A.; Eleuch, H. Coherence and information dynamics of a $\Lambda$-type three-level atom interacting with a damped cavity field. Eur. Phys. J. Plus 132, (2017), 75.

[39] Obada, A.-S.F., and Khalil, E. M. "Generation and some non-classical properties of a finite dimensional pair coherent state." Opt. Commun., 260,(2006), 19-24.

[40] Vieira, V.R.; Sacramento, P.D. Generalized Phase-Space Representatives of Spin-J Operators in Terms of Bloch Coherent States. Ann. Phys.,242, (1995), 188.

[41] Zyczkowski, K. Localization of Eigenstates \& Mean Wehrl Entropy. Physica E 9, (2001), 583.

[42] Mohamed, A.-B. A., Eleuch,H.,"Quasi-probability information in an coupled two-qubit system interacting non-linearly with a coherent cavity under intrinsic decoherence" Sci. Rep. 10 (2020) 13240.

[43] van Enk, S.J.; Kimble, H.J. Quantum Information Processing in Cavity-QED. Quant. Inform. Comput., 3 (2004): 75-90

[44] T. Yu, J. H. Eberly, Sudden Death of Entanglement. Science 323, (2009) 598.

[45] A.-B. A. Mohamed, Non-local correlations via Wigner-Yanase skew information in two SC-qubit having mutual interaction under phase decoherence. Eur. Phys. J. D 71 (2017) 261. 
Figures
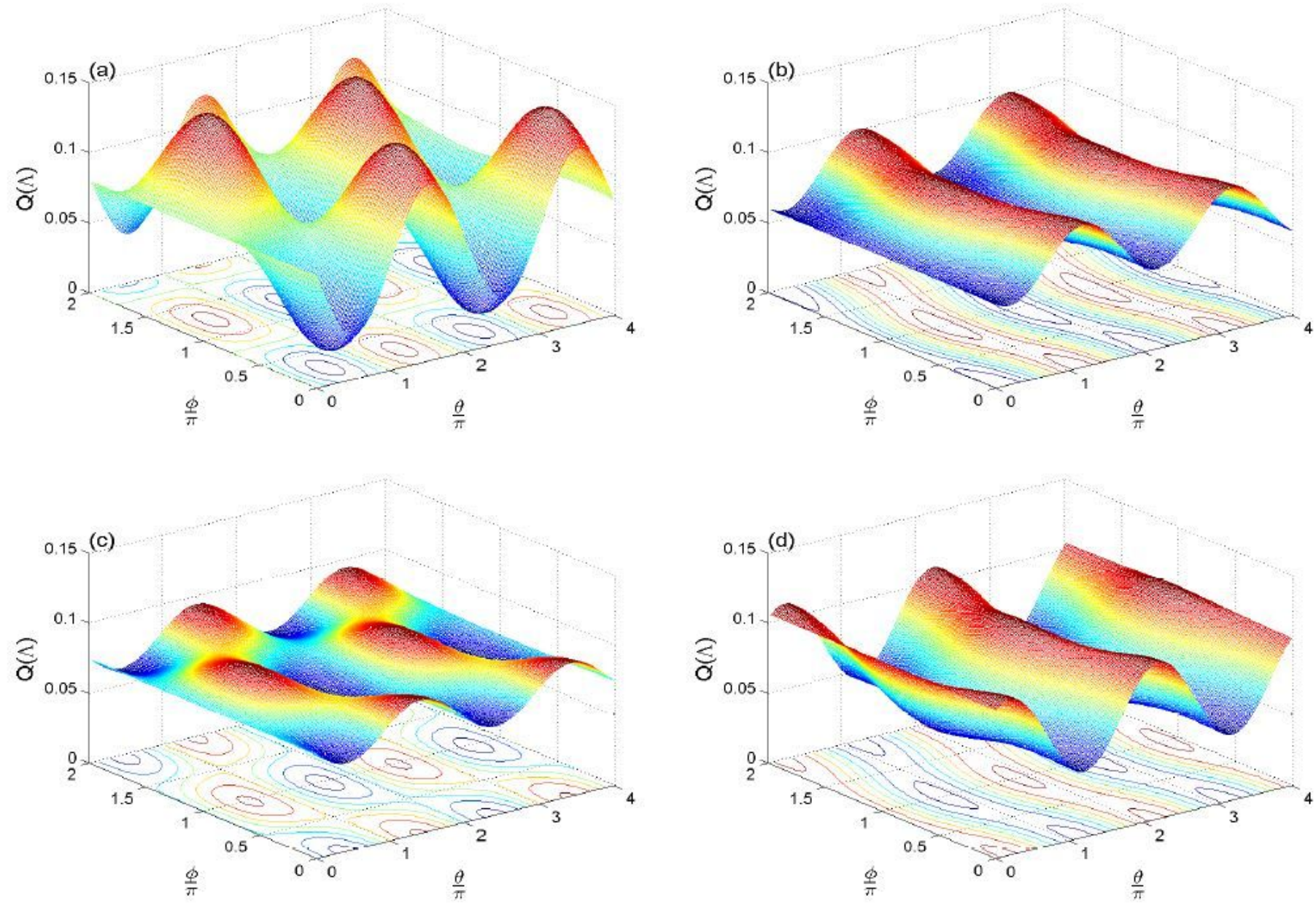

\section{Figure 1}

The Q-Husimi function at $\lambda t=0: 6 \pi$ (that corresponds to a WE minimum value) in (a) and at $\lambda t=\pi$ (that corresponds to a WE maximum value) in (b) for $\xi=2, q=40$, and $a=\beta=0$ with $\$=\pi$. In (c) and (d), we take the same data of $(a, b)$ but for $q=10$. 

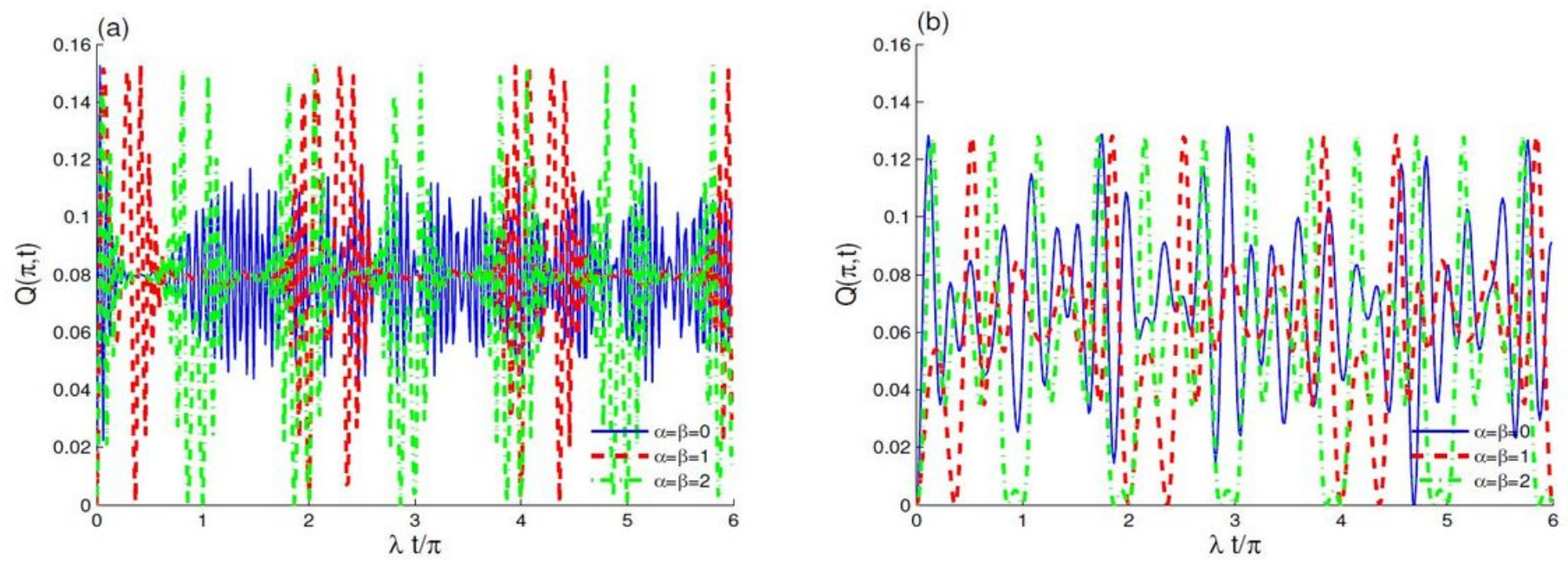

Figure 2

Time evolution of $Q(\pi ; t)$ for $\xi=2, \$=\pi$ and the different values of $(\alpha ; \beta)$ with $q=40$ in $(a)$ and $q=10$ in (b).
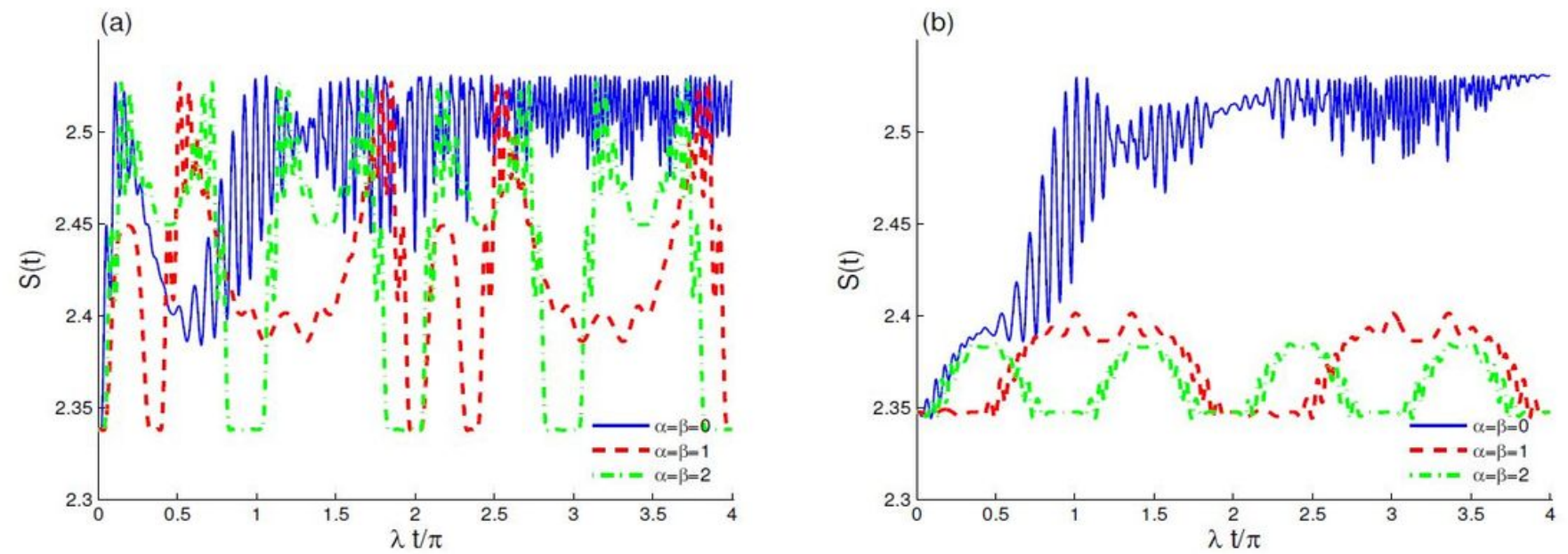

Figure 3

Dynamics of entropy $S(t)$ for $\xi=2, q=40$ and the different values of $(a ; \beta)$ with $\$=\pi$ in $(a)$ and $\$=\pi / 4$ in (b). 
(a)

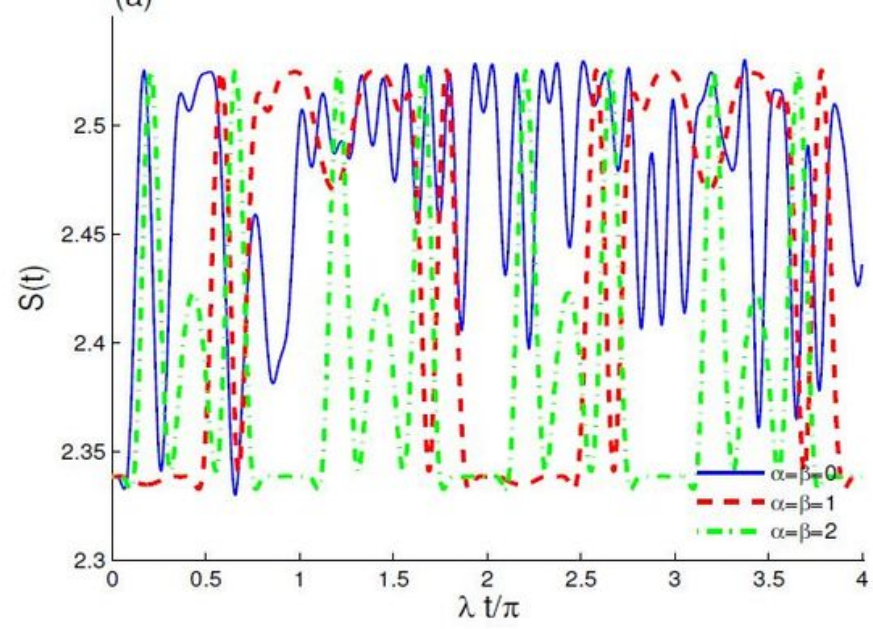

Figure 4

The same as in Fig.3 but for $q=10$.

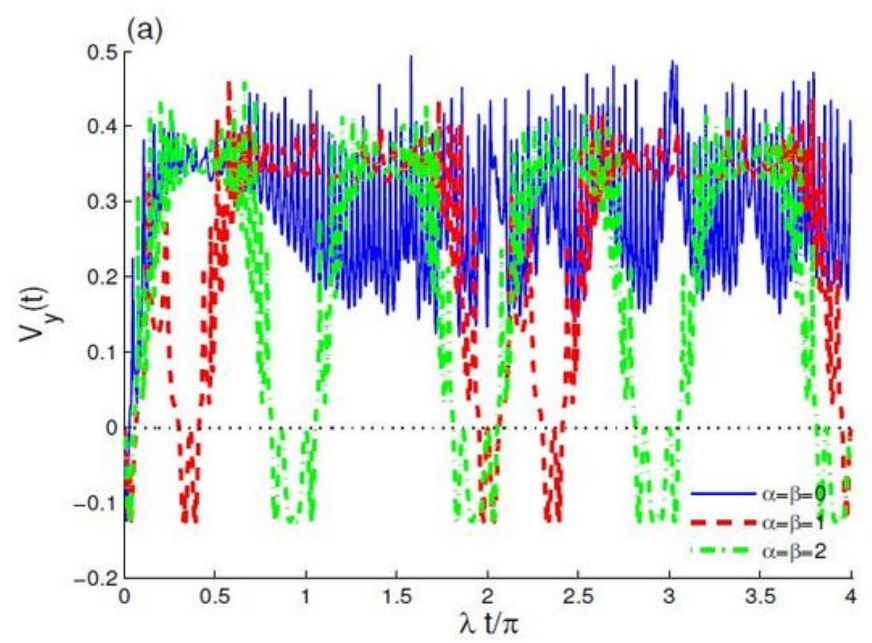

(b)
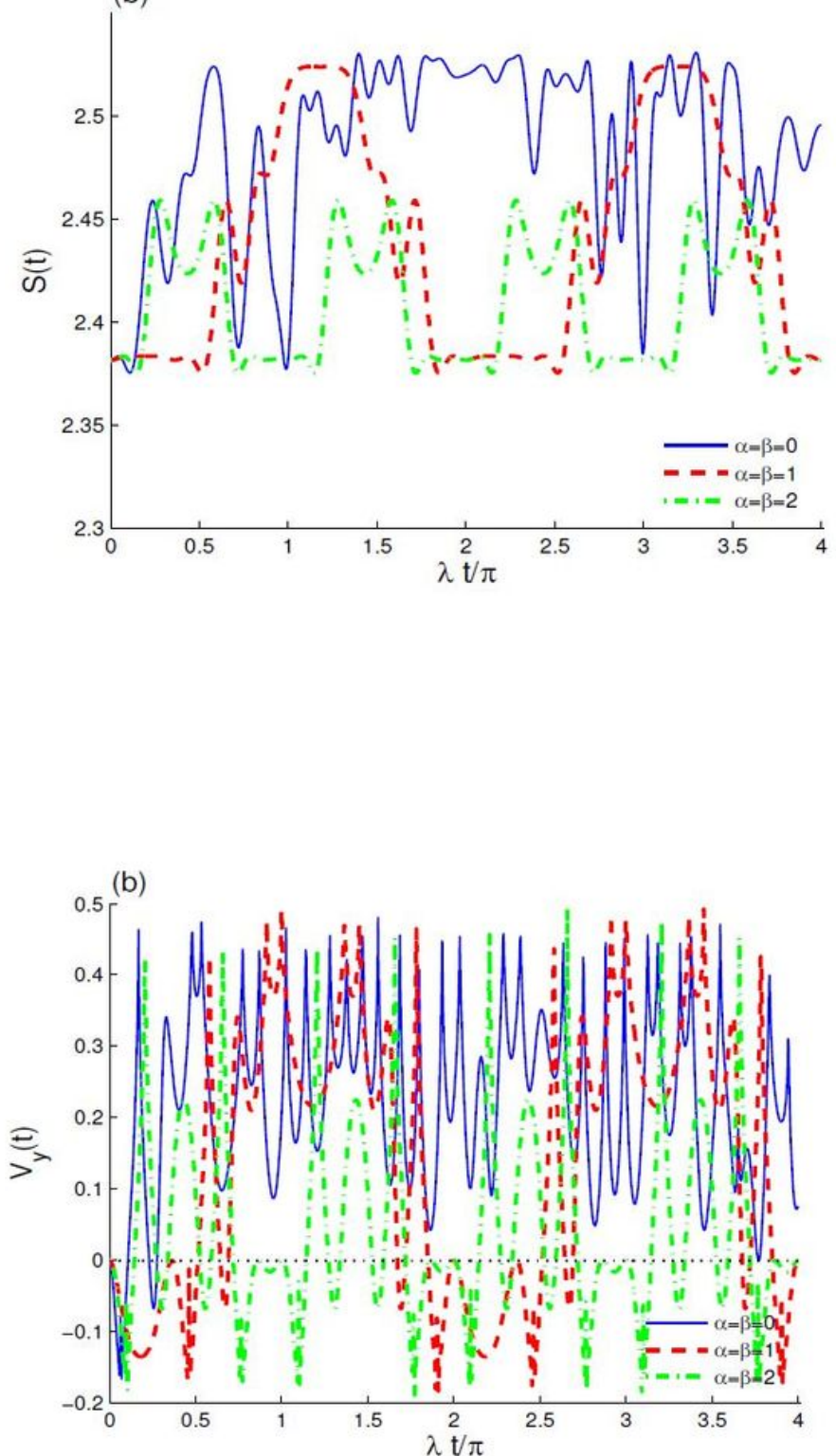

Figure 5

The variance squeezing $V y(t)$ for $\xi=2, \$=\pi$ and the different values of $(a ; \beta)$ with $q=40$ in $(a)$ and $q=10$ in (b). 

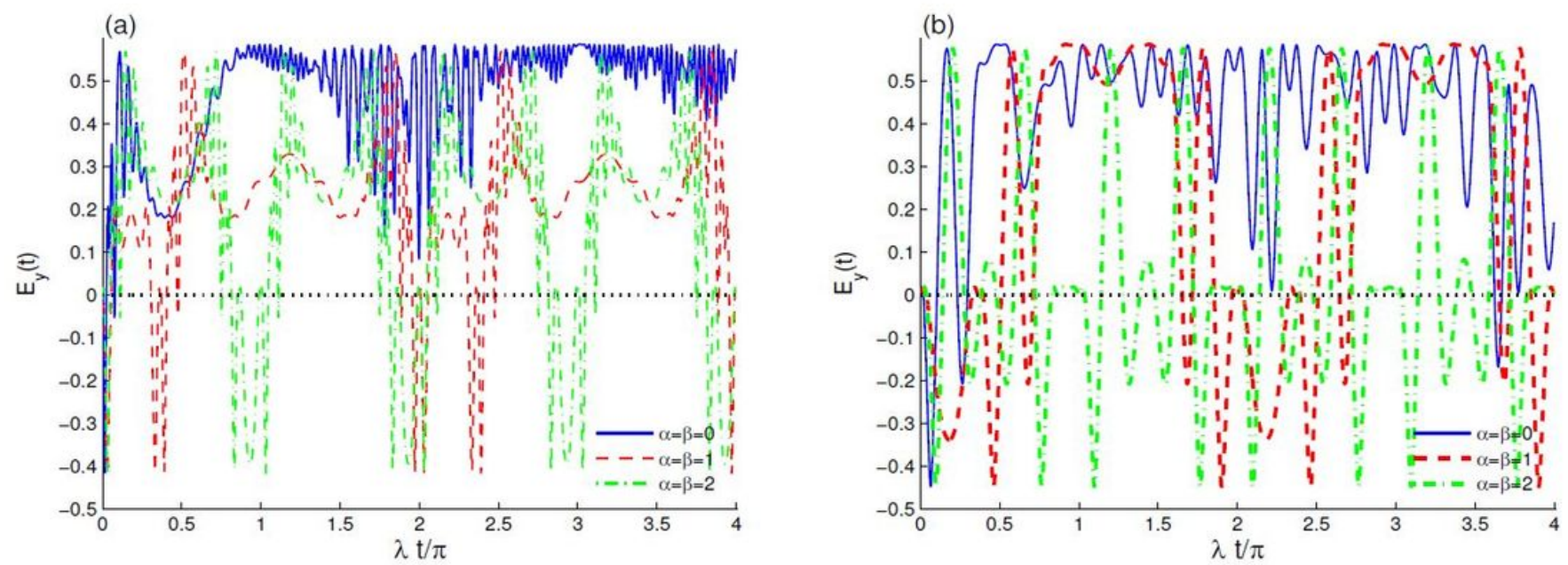

Figure 6

Dynamics of the entropy squeezing Ey(t) for $\xi=2, \$=\pi$ and the different values of $(a ; \beta)$ with $q=40$ in (a) and $q=10$ in (b).

(a)

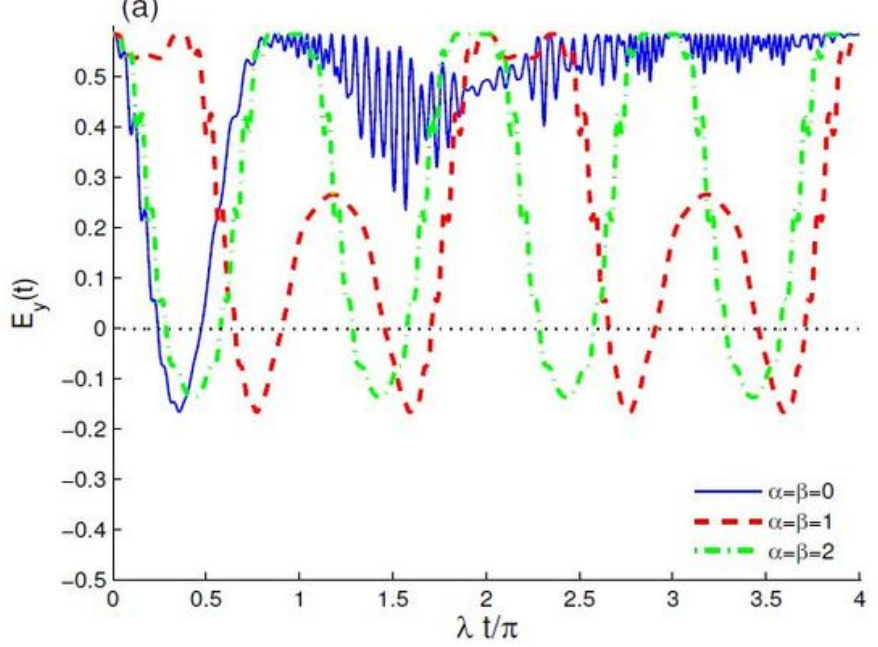

(b)

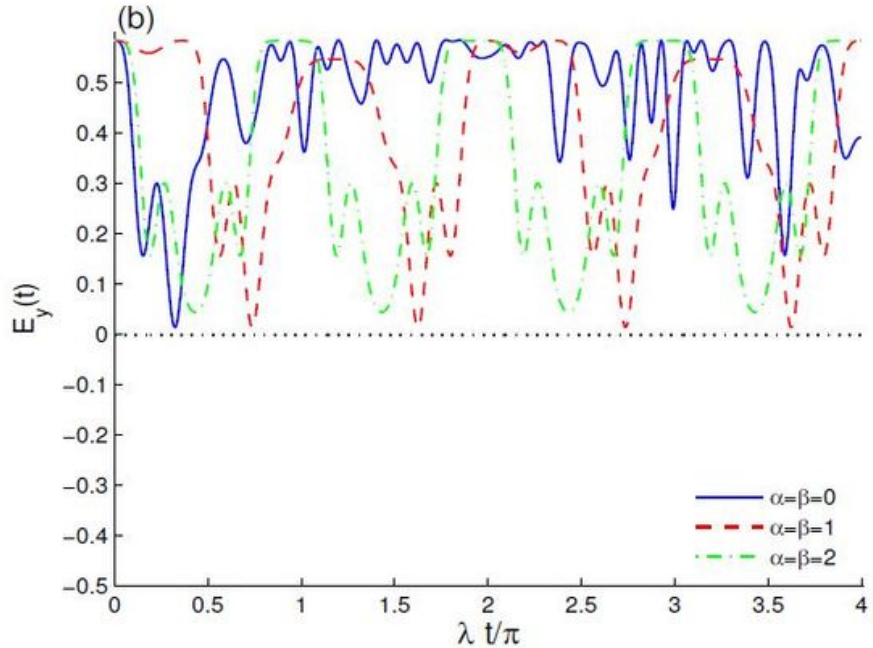

Figure 7

The same as in Fig.3 but for $\$=\pi / 4$. 